\title{
THE GAS-TO-DUST RATIO IN THE GALAXY
}

\author{
F. J. KERR and G. R. K NAPP \\ University of Maryland, College Park, Md., U.S.A.
}

\begin{abstract}
We have investigated the gas-to-dust ratio in the Galaxy by comparing $21-\mathrm{cm} \mathrm{HI}$ column densities with the color excesses of globular clusters. We find a constant gas-to-reddening ratio in interstellar clouds and the intercloud medium. This ratio is also independent of galactic latitude.
\end{abstract}

The gas-to-dust ratio in our Galaxy can be investigated observationally by comparing the visual absorption and the neutral hydrogen column density along convenient lines of sight. This has been done for RR Lyrae color excesses and $21-\mathrm{cm}$ $\mathrm{H}$ I column densities by Sturch (1969) and for OB star color excesses and $\mathrm{H}$ I Ly- $\alpha$ absorption by Savage and Jenkins (1972).

We have extended this method by measuring $21-\mathrm{cm}$ profiles in the directions of 81 globular clusters for which color excesses are available [mainly from van den Bergh (1967)] with the NRAO* 140-ft telescope.

Globular clusters are halo objects and they can be considered to be outside the layer of interstellar matter, so that all the $\mathrm{H}$ I seen along the line of sight is in front of the cluster (except in a very few low-latitude cases). Also, the sampling geometries for the color excess and $21-\mathrm{cm}$ column density data are almost identical, the globular clusters typically being comparable in angular size to the beamwidth of the telescope.

The $\mathrm{H}$ I profiles were corrected for any obvious self-absorption effects (although these only occurred for a few low-latitude clusters), and the integrated brightness $B_{\mathrm{HI}}$ calculated from:

$$
B_{\mathrm{H}_{\mathrm{I}}}=1.823 \times 10^{18} \int T_{\mathrm{b}}(v) \mathrm{d} v,
$$

where $T_{\mathrm{b}}(v)$ is the measured $\mathrm{H} \mathrm{I}$ brightness temperature at velocity $v$. If the $\mathrm{H}$ I optical depth is small at all velocities, $B_{\mathrm{HI}_{\mathrm{I}}}$ is numerically equal to $N_{\mathrm{H} I}$, the $\mathrm{H}$ I column density in atom $\mathrm{cm}^{-2}$.

A plot of $B_{\mathrm{H}_{1}}$ vs $E_{B-V}$ is shown in Figure 1. For low values of $B_{\mathrm{H}_{I}}$ and $E_{\mathrm{B}-V}\left(B_{\mathrm{H}_{1}} \lesssim\right.$ $\left.\$ 40 \times 10^{20}, E_{B-V} \lesssim 0^{m} 5\right)$ the points lie close to the straight-line relationship:

$$
B_{\mathrm{HI}_{\mathrm{I}}}=\left[(51.4 \pm 5.2) E_{\mathrm{B}-\mathrm{V}}-(0.1 \pm 1.9)\right] \times 10^{20} \mathrm{mag}^{-1} \text {. }
$$

This relationship was calculated by least squares, with errors in both coordinates. The slope corresponds to a gas: dust ratio by mass of $\sim 100: 1$ and a mean visual absorption coefficient for the interstellar medium of $\kappa_{\mathrm{v}} \sim 300 \mathrm{gm}^{-1} \mathrm{~cm}^{2}$ (see Knapp et al., 1973).

Equation (1) shows that, to well within the uncertainties, the straight-line relation-

\footnotetext{
* The National Radio Astronomy Observatory is operated by Associated Universities Inc., under contract
} with the National Science Foundation.

F. J. Kerr and S. C. Simonson, III (eds.), Galactic Radio Astronomv, 179-182. All Rights Reserved.

Copyright C 1974 by the IAU. 
ship goes through zero, i.e., the gas and dust are well mixed throughout the interstellar medium, and dust exists in the intercloud medium.

We examined our data for latitude effects, since the intrinsic luminosity of globular clusters allows us to observe at very low latitudes. Statistically, we found a very good correlation between $B_{\mathrm{HI}_{\mathrm{I}}} \sin b$ and $E_{\mathrm{B}-V} \sin b$. The ratio $E_{B-V} / B_{\mathrm{H}_{I}}$ averaged over each $10^{\circ}$ of latitude is shown in Figure 2 ; it can be seen that any deviations from a con-

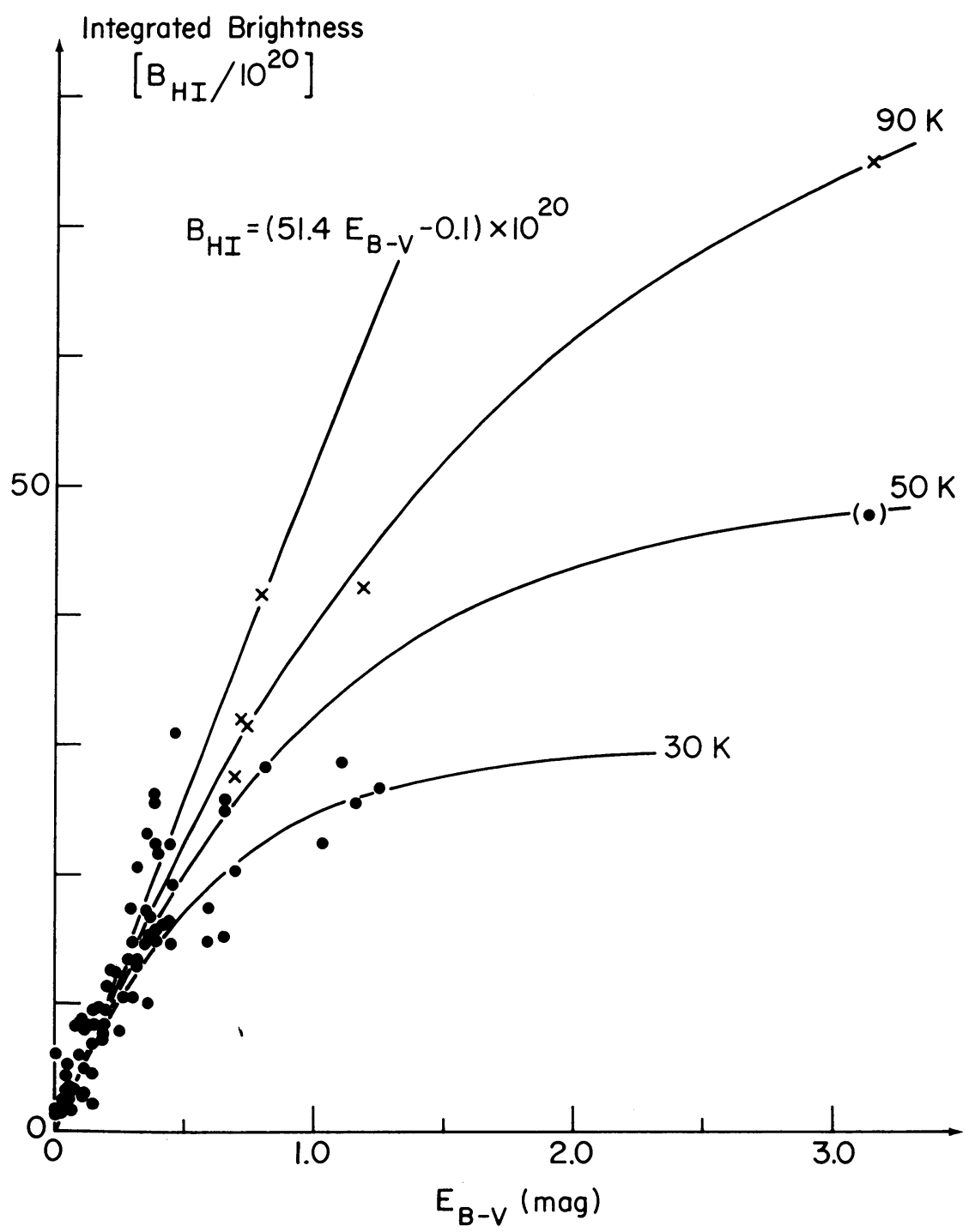

Fig. 1. Plot of $B_{\mathrm{H}_{\mathrm{I}}}$ vs $E_{\mathrm{B}-V}$ for 81 globulars. The crosses correspond to points corrected for self-absorption. The uncorrected point for the highly reddened cluster Terzan 5 is included in parentheses. The straight line relationship is as defined in Equation (2); the curves represent the $B_{\mathrm{H}_{I}}-E_{B-V}$ relationship assuming a constant value for $N_{\mathrm{HI}} / E_{B-V}$ and various values of the harmonic mean spin temperature of the $\mathrm{HI}_{\mathrm{I}}$. 
stant ratio are not significant. These results imply that the gas and dust have the same scale height.

The scatter of points with high values of $E_{B-V}$ and $B_{\mathrm{H} I}$ to the high color excess side of the mean relationship shown in Figure 1 may be due mainly to a combination of saturation and molecule formation. In Figure 1 we also show curves corresponding

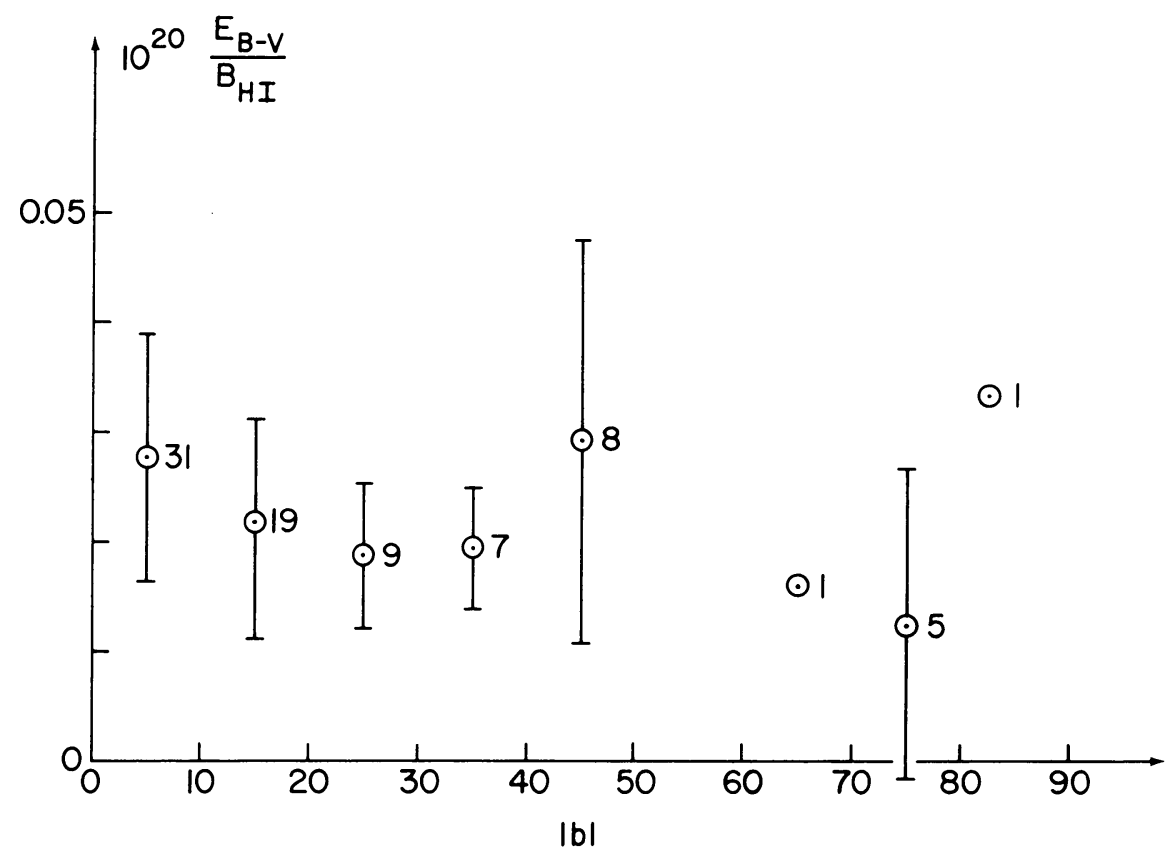

Fig. 2. Plot of $E_{B-V} / B_{\mathrm{H}_{\mathrm{I}}}$ averaged over $10^{\circ}$ latitude intervals. The number beside each point corresponds to the number of individual observations; the error bars represent the rms deviation of each sample.

to various $\mathrm{H}$ I spin temperatures and a gas-to-dust ratio as given by Equation (1), and it can be seen that saturation of the $\mathrm{H}_{\mathrm{I}}$ can easily explain much of the scatter. Points with particularly low values of $B_{\mathrm{HI}} / E_{B-V}$ were all found to correspond to clusters lying behind discrete dense dust clouds, so that some of the scatter is probably also due to the formation of hydrogen molecules.

In summary, we find that the gas and dust in the Galaxy are well mixed both in and out of clouds, and that the gas-to-dust ratio is independent of latitude. Apparent departures are due to a combination of saturation of the gas and the formation of $\mathrm{H}_{2}$.

\section{Acknowledgements}

We thank the NRAO for the observing time for this project. This research is supported by the U.S. National Science Foundation. 


\title{
References
}

Knapp, G. R., Kerr, F. J., and Rose, W. K.: 1973, Astrophys. Letters 14, 187.

Savage, B. D. and Jenkins, E. B.: 1972, Astrophys. J. 172, 491.

Sturch, C.: 1969, Astron. J. 74, 82.

van den Bergh, S.: Astron. J. 72, 70.

\author{
F. J. Kerr \\ G. R. Knapp \\ Astronomy Program, University of Maryland \\ College Park, Md. 20742, U.S.A.
}

\section{DISCUSSION}

Jenkins: One should not underestimate the importance of $\mathrm{H}_{2}$ formation in causing a significant straggling of points below your correlation line when color excesses are large. In our most recent study of the gas and dust correlation from OAO-2 observations of $\mathrm{Ly}-\alpha$ absorption, Savage and 1 found a significant improvement in the relation if we replaced $n\left(\mathrm{HI}_{\mathrm{I}}\right)$ with $n(\mathrm{HI})+2 n\left(\mathrm{H}_{2}\right)$ for those stars for which $\mathrm{H}_{2}$ column densities were available from Copernicus observations.

Kerr: The radio observations cannot easily distinguish between saturation and molecule formation in individual cases. Therefore, your ultraviolet observations are very important.

Oort: How were the color excesses of the clusters obtained? How did you get the normal colors?

Kerr: These were taken from van den Bergh's list, mainly.

Shuter: In your plot of $E_{B-V}$ vs $N_{H_{1}}$ is it not true that departures from linearity at large $E_{B-V}$ occur for observations at low galactic latitudes or long galactic paths and are therefore more likely to be due to saturation in the HI profiles rather than by molecule formation.

$K e r r$ : In general, yes, but there are cases at quite low galactic latitudes where $E_{B-V}$ is low - for example, Baade's famous 'window' near the direction of the center.

Greenberg: How do you know for certain that $\mathrm{H} \mathrm{I}$ and dust are in the same region along the line of sight?

Kerr: This is not necessary, as long as the globulars are outside the layer of interstellar material. In that case, it is only the integral that counts. There are however differences in solid angle between the radio beam and the cluster size, which may sometimes have an effect.

Radhakrishnan: You stated that you felt that gas and dust were well mixed in both cloud and intercloud regions. What is the evidence for dust in the intercloud medium?

Kerr: It is the fact that the straight line giving the mean relationship between the gas column density and the color excess passes through the zero point, implying that all gas has dust associated with it. Some $21-\mathrm{cm}$ emission can be seen at all points in the sky, whereas the reported color excess is sometimes zero, but this is because the $21-\mathrm{cm}$ measurement is more sensitive.

Baldwin: Is the variation in $N_{\mathrm{H}}$ from point to point mainly due to the influence of galactic latitude? If so, it is surely difficult to be sure that the scale height of the gas and dust are really the same.

Kerr: We examined the data carefully, and have shown that the ratio of gas column density to color excess is the same, irrespective of latitude. 